\title{
Dynamic Updating of Working Memory Resources for Visual Objects
}

\author{
Nikos Gorgoraptis, Raquel F. G. Catalao, Paul M. Bays, and Masud Husain \\ Institute of Cognitive Neuroscience and Institute of Neurology, University College London, London WC1N 3AR, United Kingdom
}

Recent neurophysiological and imaging studies have investigated how neural representations underlying working memory (WM) are dynamically updated for objects presented sequentially. Although such studies implicate information encoded in oscillatory activity across distributed brain networks, interpretation of findings depends crucially on the underlying conceptual model of how memory resources are distributed.

Here, we quantify the fidelity of human memory for sequences of colored stimuli of different orientation. The precision with which each orientation was recalled declined with increases in total memory load, but also depended on when in the sequence it appeared. When one item was prioritized, its recall was enhanced, but with corresponding decrements in precision for other objects. Comparison with the same number of items presented simultaneously revealed an additional performance cost for sequential display that could not be explained by temporal decay. Memory precision was lower for sequential compared with simultaneous presentation, even when each item in the sequence was presented at a different location.

Importantly, stochastic modeling established this cost for sequential display was due to misbinding object features (color and orientation). These results support the view that WM resources can be dynamically and flexibly updated as new items have to be stored, but redistribution of resources with the addition of new items is associated with misbinding object features, providing important constraints and a framework for interpreting neural data.

\section{Introduction}

One of the fundamental properties of working memory (WM) is its limited capacity (Cowan, 2001; Baddeley, 2003). For vision, this has been estimated to be three or four items, based on the ability of observers to detect changes made to a static array of objects over a brief delay (Phillips, 1974; Pashler, 1988; Luck and Vogel, 1997; Vogel et al., 2001; Todd and Marois, 2004). But in real-world situations, the visual input to the brain is constantly changing with movements of the body and alterations in the environment, so ecologically important objects are often viewed in sequence. Vital cognitive processes - such as action selection and planning - therefore have to be informed by memory for objects that have been replaced by others. The neural mechanisms involved in maintaining these representations across intervening items and over time have become the focus of intense investigation using neurophysiological and imaging techniques (D’Esposito et al., 1999; Marshuetz et al., 2000; Xu and Chun,

\footnotetext{
Received Jan. 12, 2011; revised April 26, 2011; accepted April 29, 2011.

Author contributions: N.G., P.M.B., and M.H. designed research; N.G. and R.F.G.C. performed research; P.M.B. contributed unpublished reagents/analytic tools; N.G. analyzed data; N.G. and M.H. wrote the paper.

This work was supported by the Wellcome Trust and the National Institute of Health Research Comprehensive Biomedical Research Centre at University College London Hospital/University College London. N.G. was supported by a graduate scholarship from the Greek State Scholarships Foundation. We thank Dr Yoni Pertzov and the anonymous reviewers for useful comments and suggestions.

The authors declare no competing financial interests.

Correspondence should be addressed to Dr. Nikos Gorgoraptis, Institute of Cognitive Neuroscience and Institute of Neurology, University College London, 17 Queen Square, London WC13AR, UK. E-mail:n.gorgoraptis@ucl.ac.uk. DOI:10.1523/JNEUROSCI.0208-11.2011

Copyright $\odot 2011$ the authors $\quad 0270-6474 / 11 / 318502-10 \$ 15.00 / 0$
}

2006; Siegel et al., 2009; Jenkins and Ranganath, 2010; Takahama et al., 2010; Warden and Miller, 2010).

Like change detection experiments, studies examining visual WM for serially presented items have tested recall in a binary fashion, assuming that each object in a sequence is either perfectly stored or entirely forgotten (Phillips and Christie, 1977; Smyth et al., 2005; Johnson and Miles, 2009). But this approach does not provide any information on the fidelity of stored representations. An alternative method is to measure the variability of memory estimates around the true value, i.e., the precision of recall for object attributes such as location, orientation, and color (Palmer, 1990; Wilken and Ma, 2004; Bays and Husain, 2008; Bays et al., 2009, 2011; Fougnie et al., 2010).

This approach has recently prompted a reevaluation of the classical view of visual WM as comprising a fixed number of slots, each maintaining a single object with high resolution. Instead, such investigations have led to a radically different proposal: that although WM resources are highly limited, they are not quantized, so they can be flexibly distributed to prioritize a few items for high resolution storage or store a larger number with lower fidelity (Alvarez and Cavanagh, 2004; Wilken and Ma, 2004; Bays and Husain, 2008; Bays et al., 2009).

This is a fundamentally different conceptual framework to the traditional account and has obvious, important implications for research that seeks to examine the neural basis of WM. However, previous studies have not examined the precision of memory when objects are presented sequentially. Here we compared memory resolution for objects in sequences with simultaneously presented items and used a probabilistic model to account for the 


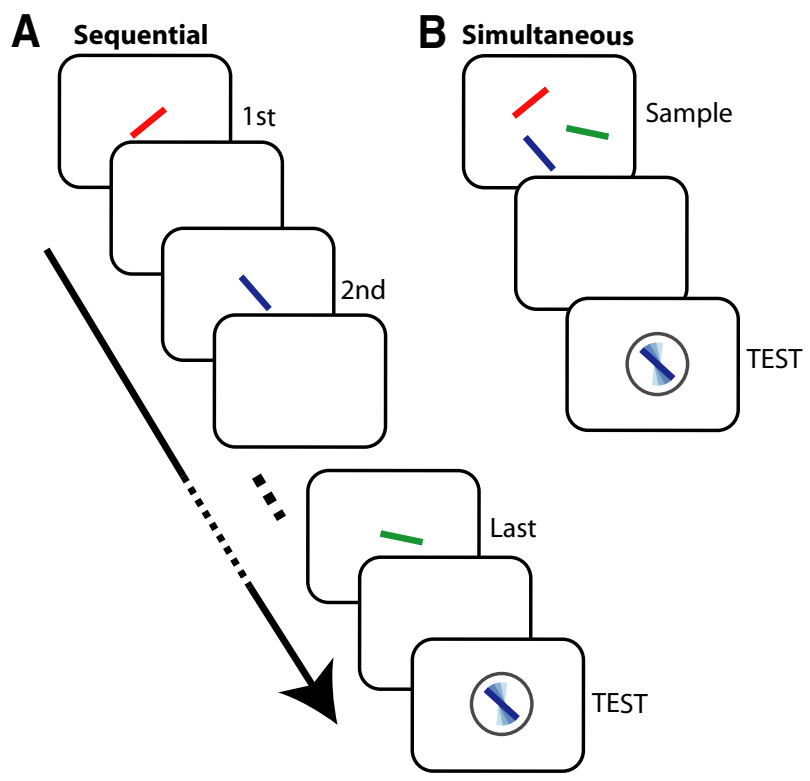

Figure 1. Experimental paradigm for sequential and simultaneous tasks. $A$, In experiment 1 , participants were presented with a sequence of colored bars, each with a different orientation. A probe item of a randomly chosen color (in this case, blue) was then presented and subjects adjusted the orientation of the probe item to that of the orientation of the item of the same color shown in the sequence (in this case, the second item). $\boldsymbol{B}$, In experiment 2 , stimuli were shown simultaneously, in an array, following which the orientation of one of the items (in this case, blue) was probed and had to be reproduced from memory.

distribution of responses. We demonstrate how memory precision changes as new items are presented and how resources are flexibly weighted to prioritize task-relevant items. Importantly, we find that memory for sequentially presented objects is especially prone to corruption by features belonging to other items in the sequence (misbinding). These observations provide important constraints on the neural mechanisms underlying WM for objects encoded sequentially by the visual system over time.

\section{Materials and Methods}

\section{Participants}

A total of 34 healthy volunteers (19 female, 15 male, age: 19-34 years) participated in the study after providing written informed consent to procedures approved by the local ethics committee. All participants had normal or corrected-to-normal visual acuity and reported normal color vision. Nine volunteers [six female, age: $25.8 \pm 5.3$ years (mean \pm SD)] took part in experiment 1 , eight volunteers (six female, age: $21.4 \pm 3.1$ ) participated in experiment 2 , eight (four female, $24.8 \pm 2.5$ ) in experiment 3 , and nine (three female, age: $25 \pm 2.7$ ) in experiment 4 .

\section{Experiment procedure}

Experiment 1: Sequential presentation. A schematic representation of the task is shown in Figure $1 \mathrm{~A}$. Each trial consisted of a sequence of one to five colored bars $\left(2^{\circ} \times 0.3^{\circ}\right.$ of visual angle $)$ consecutively presented on a gray background on a 21 inch CRT monitor at a viewing distance of 60 $\mathrm{cm}$. Each bar had a different color and orientation and all were presented at fixation, at the center of the display. The sequence of colors in each trial was produced by permutation of a random selection of five easily distinguishable colors. On each trial, participants did not know in advance how many objects they would have to remember. Stimuli within the same sequence differed by at least $10^{\circ}$ in orientation, which was otherwise random. Each stimulus was shown for $500 \mathrm{~ms}$, followed by a blank screen for $500 \mathrm{~ms}$.

At the end of each sequence, recall for one of the items was probed by redisplaying a bar of the same color with a random orientation. A circle surrounding this probe item made it easily distinguishable from the tobe-remembered items in the sequence. Subjects were instructed to rotate the probe using a response dial (Logitech) to match the remembered orientation of the item of the same color in the sequence-henceforth termed the target. Note that we use the term "target" here simply to distinguish from other objects in the sequence, or nontargets, that were not probed. We emphasize that in this experiment, participants did not know which item would be tested or how long each sequence would be from trial-to-trial.

Each subject completed a total of 400 interleaved trials. There were 25 trials for each of the 15 combinations of sequence length (1-5) and serial position of the target item within the sequence ( 375 trials in total). In addition, there were 25 trials where a single item was followed by a longer blank period of $3500 \mathrm{~ms}$ (equivalent in duration to a four-item sequence). These trials were presented interleaved with other conditions to examine the pure effects of temporal delay on memory in the absence of intervening objects.

Experiment 2: Simultaneous presentation. To compare WM precision for items presented sequentially with objects displayed simultaneously, we ran the task shown in Figure $1 B$. Each trial started with a central fixation cross displayed on a gray background. Once stable fixation was established, one to five bars, each of a different color and orientation, were presented simultaneously. The display settings, dimensions of the stimuli, and the selection of color and orientation were as in experiment 1. Stimuli were displayed at random positions on an invisible circle of radius $6^{\circ}$, with a minimum center-to-center separation of $3^{\circ}$ of visual angle. This memory array was shown for $1000 \mathrm{~ms}$, followed by a $1000 \mathrm{~ms}$ blank screen. Subsequently, one of the items in the array was probed by color at central fixation and the participant had to indicate the remembered orientation of the item, as in experiment 1 . Each subject completed 500 trials. Eye position was monitored online at $1000 \mathrm{~Hz}$ using a framemounted infrared eye tracker (SR Research) to ensure subjects maintained central fixation. Trials were repeated if gaze deviated $>2^{\circ}$ from the fixation cross during stimulus presentation.

Experiment 3: Simultaneous versus sequential presentation at different locations. In experiment 1 , items were presented at the same spatial location, which may confound the comparison with simultaneous presentation, where each item occupied a different location in space. Therefore, we performed an additional experiment to distinguish the effects of sequential versus simultaneous presentation from the potentially confounding effects of presenting stimuli at the same or at different locations. In experiment 3, two, four, or six items were presented either simultaneously or sequentially, always at different locations, with a minimum center-to-center separation of $3^{\circ}$ of visual angle. All stimuli were presented at an eccentricity of $6^{\circ}$ for $500 \mathrm{~ms}$, followed by a $500 \mathrm{~ms}$ blank screen. The dimensions of the stimuli and the selection of color and orientation were identical to the previous experiments. As in experiment 2 , central fixation was monitored online using infrared eye tracking and trials were repeated if gaze deviated $>2^{\circ}$ from the fixation cross during stimulus presentation. At the end of each trial, one of the items was probed by color at the center of the screen, as in the previous experiments. Each subject completed a total of 480 trials consisting of four 60 -trial blocks of sequential and four blocks of simultaneous presentation. The order of the eight blocks was randomized.

Experiment 4: Prioritizing one of the items in a sequence. To investigate how WM resources are allocated to a prioritized (cued) item in a sequence compared with noncued objects, we investigated performance on a variant of experiment 1 . Here, a sequence of four items was presented on each trial, using the same display settings, stimulus dimensions, and durations as in experiment 1 . Participants were instructed before the experiment that items of one specified color were more likely to be tested. This cue color was different for each subject and fixed throughout each experimental session. There were two experimental conditions: the cue present condition and the baseline condition. In the cue present condition, one of the four items on each trial was of the cue color. This item was probed on a higher proportion of trials $(62.5 \%$, as opposed to $12.5 \%$ for each of the other three items). In the baseline condition, the cue color was not present in the sequence and all items were equally likely to be probed (25\%), and therefore equally task-relevant. The participant had to indicate the remembered orientation of the target item using a dial to rotate the probe bar, as in the previous experiments. Each subject completed a 
total of 300 trials, consisting of four blocks of 50 trials for the cue present condition and two blocks of 50 trials for the baseline condition. The order of the blocks was randomized.

\section{Analysis}

For each trial, a measure of error was obtained by calculating the angular deviation between the orientation reported by the subject and the correct orientation of the target bar in the preceding sequence. Precision was calculated as the reciprocal of the SD of error across trials $(1 / \sigma)$. As the parameter space for orientation is circular, we used Fisher's definition of SD for circular data (Fisher, 1993), subtracting the value expected for chance; therefore, a precision value of zero corresponds to responding at random. This method of estimating the fidelity of recall of a visual stimulus based on the distribution of error has previously been described for orientation, location (Bays and Husain, 2008), and color (Bays et al., 2009), but only for simultaneous displays where all objects to be remembered were presented together.

Precision was calculated separately for each subject, set size and condition. Hypotheses regarding the effects of experimental parameters (number of items, order in sequence, cueing condition) on precision were tested by ANOVA and $t$ tests (see Results, below).

Additionally, to assess the effects of prioritizing items (cued objects) at different serial positions in experiment 4, we calculated, for each serial position in the sequence, the fractional difference in precision between the cue present and baseline conditions as $\left(P_{\mathrm{C}}-P_{\mathrm{B}}\right) /\left(P_{\mathrm{C}}+P_{\mathrm{B}}\right)$, where $P_{\mathrm{C}}$ is the precision in a cue present sequence and $P_{\mathrm{B}}$ the precision in a baseline sequence. For this analysis, we pooled data across subjects, increasing the number of trials on which each precision calculation was based.

To quantify the contribution of different sources of error to overall precision estimates in each experiment, we applied a probabilistic model introduced previously by Bays et al. (2009). This model, building on an earlier proposal by Zhang and Luck (2008), attributes errors on the reproduction task to three sources, as follows: (1) Gaussian variability in memory for the target orientation; (2) a certain probability on each trial of misreporting one of the other nontarget orientations in the sequence; and (3) a certain probability of responding with a random orientation not related to any of the items in the sequence. This model is described as follows:

$$
p(\hat{\theta})=a \phi_{K}(\hat{\theta}-\theta)+\beta \frac{1}{m} \sum_{i}^{m} \phi_{K}\left(\hat{\theta}-\varphi_{i}\right)+\gamma \frac{1}{2 \pi}
$$

where $\theta$ is the true orientation of the target item, $\hat{\theta}$ the orientation reported by the subject, and $\phi_{\kappa}$ is the von Mises distribution (the circular analog of the Gaussian) with mean zero and concentration parameter $\kappa$. The probability of reporting the correct target item is given by $\alpha$. The probability of mistakenly reporting a nontarget item is given by $\beta$, and $\left\{\varphi_{1}, \varphi_{2}, \ldots \varphi_{\mathrm{m}}\right\}$ are the orientations of the $m$ nontarget items. The probability of responding randomly is given by $\gamma=1-\alpha-\beta$. A graphical representation of these model components is given in Figure 5.

Maximum likelihood estimates (Myung, 2003) of the parameters $\kappa, \alpha, \beta$, and $\gamma$ were obtained separately for each subject and set size in experiments 1 , 2 and 3, using an expectation-maximization algorithm (MATLAB code available at http://www.sobell.ion.ucl.ac.uk/pbays/code/JV10/).

To investigate how serial order of the target item in a sequence affected the model parameters, we also fit the model separately for each combination of serial position and sequence length in experiment 1 . As this meant dividing the data from each subject between a large number of conditions, we pooled across subjects, maximizing the data available for each condition. Similarly, in experiment 4 , we fit the model separately for trials on which cued and uncued items were probed in the cue present condition and for all items in the baseline condition, again pooling across subjects. Likelihood-ratio tests were used for statistical comparison of parameter values estimated from pooled data.

\section{Results}

\section{Effects of serial order and set size on recall precision}

In experiment 1 , subjects were presented with a sequence of randomly oriented colored bars and asked to reproduce from mem-

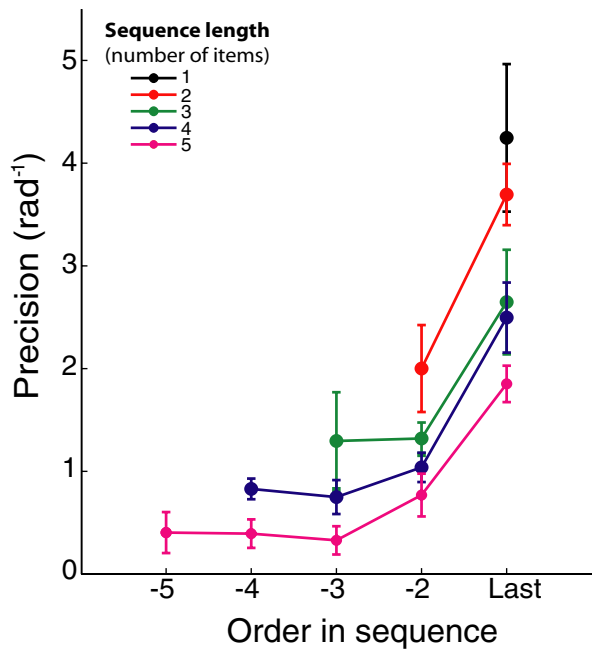

Figure 2. Serial order and sequence length modulate memory precision. Precision is plotted against order in the sequence, i.e., when in a sequence the item probed after the end of the sequence had appeared. Each colored line represents a different sequence length. The last item was remembered most accurately, while earlier items in the sequence were recalled with similar precision. Error bars represent SEM.

ory the orientation of one bar, specified by color (Fig. $1 A$ ). The total number of stimuli varied between one and five; participants were unaware of how many would be displayed in each trial. All items in the sequence were equally likely to be tested.

Figure 2 shows how the precision with which subjects recalled an item's orientation varied as a function of its serial position (i.e., when it appeared in a sequence), for different sequence lengths (denoted by different colors). Serial order had a significant effect on precision, regardless of the total number of items in the sequence (two-way ANOVA, set size $\times$ serial position, main effect of serial position: $F_{(4,120)}=11.2, p<0.001$ ) with the most recent item remembered significantly more accurately than preceding items (two-way ANOVA, simple contrast to last item: $\left.F_{(4,120)}=3.67, p=0.007\right)$. Thus, there was a clear recency effect. No statistically significant differences in precision were observed for earlier positions in a sequence (main effect of serial position with final item excluded: $\left.F_{(3,80)}=0.57, p=0.64\right)$. Performance was significantly better than chance for every combination of serial order and set size $\left(t_{(8)}>2.9, p<0.023\right)$, indicating that some information was stored about every item in a sequence.

How does the total number of objects in the sequence affect the fidelity of recall? As shown in Figure 2, when comparing sequences of different lengths, for every serial position, precision decreased significantly as the number of items increased (main effect of set size: $F_{(4,120)}=11.8, p<0.001$; set size $\times$ serial position interaction: $\left.F_{(6,120)}=0.23, p=0.97\right)$. Remarkably, this effect was present even for the last (and best remembered) item in a sequence: precision for the final item decreased significantly as the number of preceding items increased (main effect of set size, final items only: $\left.F_{(4,40)}=4.7, p=0.004\right)$. Therefore, as the total number of items held in memory increases, there is a loss of fidelity in recall of items of any serial order, including the most recent. Note that many previous studies of serial WM, using for example verbal or visuospatial lists (Broadbent and Broadbent, 1981; Burgess and Hitch, 1999; Logie et al., 2000), have also shown recency effects but, crucially, in those studies, participants were either able to report an item or not, in a binary (all or nothing) fashion. Here, we were able to show that the fidelity with which the last item is recalled is modulated in a graded manner by 


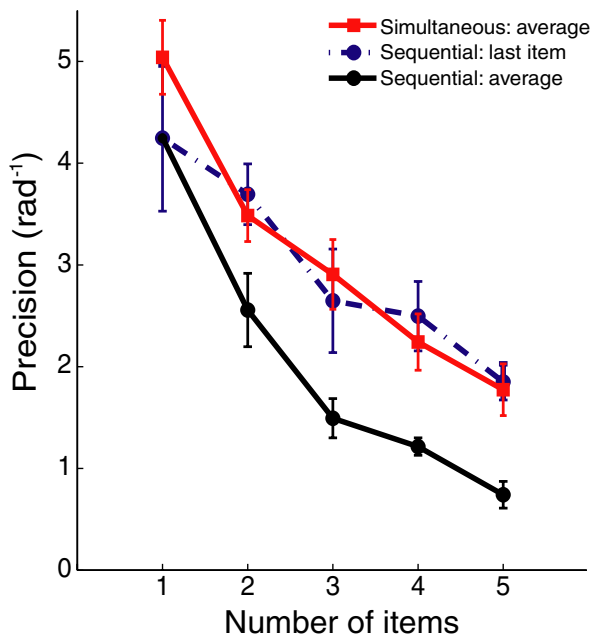

Figure 3. Effect of the number of items on memory in sequential and in simultaneous presentation. Average precision (across all items) decreased with increasing number of items presented in a sequence (black line) or simultaneously (red line). Note that the last object in a sequence (blue dashed line) was remembered with similar precision to an item in an array of the same number of simultaneously presented objects. There was no significant difference for one item in sequential compared with simultaneous conditions. Error bars are SEM.

the number of items that precede it. Such a result has important implications for the neural encoding of items in WM, as we discuss below.

\section{Comparison with simultaneous presentation}

The results of experiment 1 also have implications for models of WM because they are consistent with the principles of a shared resource model of working memory, which until now has been applied only to simultaneous displays (Wilken and Ma, 2004; Bays and Husain, 2008; Bays et al., 2009). Specifically, the findings above show that as the total number of items in memory increases, the proportion of resources dedicated to each item declines, degrading the fidelity of storage. A simple slot model limited to three objects (Luck and Vogel, 1997; Cowan, 2005) would not predict such a graded decline in performance for sequences below the capacity limit of three, since each item should be capable of being stored with equal, high resolution up to that limit. Only after all available slots had been occupied would one expect a rapid decline in precision.

From the perspective of a shared resource model, the recall advantage for the final item in a sequence we observed here could result from an uneven distribution of resources, with the largest proportion allocated to the most recently presented item. To investigate this possibility further, we compared the results of sequential displays in experiment 1 with a second task that differed only in that all items were presented simultaneously in a single display (Fig. $1 B$ ). This provides a direct comparison of how resources are allocated when information is processed sequentially in a temporal stream versus when it is presented all together to the visual system.

In Figure 3, mean recall precision is presented as a function of the total number of items presented for sequential (black symbols) and simultaneous (red symbols) display. While precision declined similarly with increasing number of items in both cases, on average, items were recalled with significantly lower precision when presented sequentially $\left(F_{(1,83)}=22.2, p<0.001\right)$. Importantly, however, this cost for sequential presentation was confined to the earlier items in each sequence.

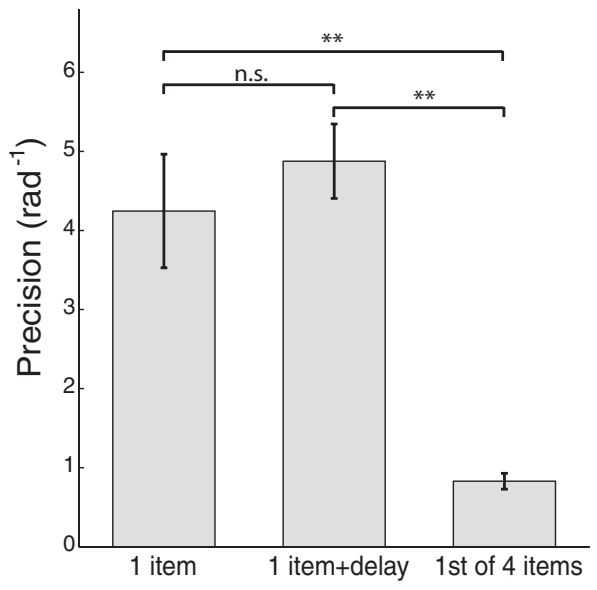

Figure 4. Loss of precision for previous items is not time-dependent. When compared with a single item (left bar), memory precision was not affected by a longer retention time (middle bar), but was significantly lower when three further items were presented in the same retention period (right bar). Error bars are SEM. ${ }^{* *} p<0.001$; n.S., nonsignificant.

When performance for only the last item in each sequence was considered (Fig. 3, blue symbols), it was found to be stored with equivalent resolution to items in a simultaneous display of the same number of items $\left(F_{(1,83)}=0.18, p=0.67\right)$, while memory for all previous items in the sequence was significantly less precise $\left(F_{(1,7)}=47.7, P<0.001\right)$. Thus, for example, if the total sequence length was three items, the last item was recalled with precision equivalent to when three items were presented simultaneously, but the previous items were recalled with significantly lower precision than the average precision for three simultaneously presented objects. Therefore, every time a new item was added to a sequence, the precision with which it was remembered was limited only by the number of previous items already stored in memory, just as for simultaneous presentation of the same number of items. However, crucially, earlier items were remembered significantly less accurately. Thus, memory precision for these is not simply determined by the total number of objects that have to be kept in memory, but is also limited by some additional source of error, which we sought to determine.

\section{Cost of sequential presentation is not due to temporal decay}

There is a long-standing controversy as to whether WM recency effects are a result of interference between remembered objects, or whether time-related memory decay also plays a role (Hole, 1996; Berman et al., 2009; Lewandowsky et al., 2009; Zhang and Luck, 2009). To control for the possibility that loss in precision for earlier items is due to time-dependent decay in memory representation, we compared precision for the first item from a sequence of four with a single object followed by a long retention period, equivalent in duration to a sequence of three further items. We observed that recall was not affected by the longer retention period $\left(t_{(8)}=1.1, p=0.32\right.$ ) (Fig. 4), but there was a fivefold decrease in memory precision when three consecutive items were presented during the same amount of time $\left(t_{(8)}=8.9\right.$, $p<0.001)$. Therefore, rather than time-related decay of their memory representation, the loss in accuracy of recall for earlier items in this study is due to the presence of the subsequent items.

\section{A probabilistic model to investigate the sources of error in sequences}

The precision measure used thus far to describe performance is a nonparametric statistic reflecting the fidelity of recall of a target 

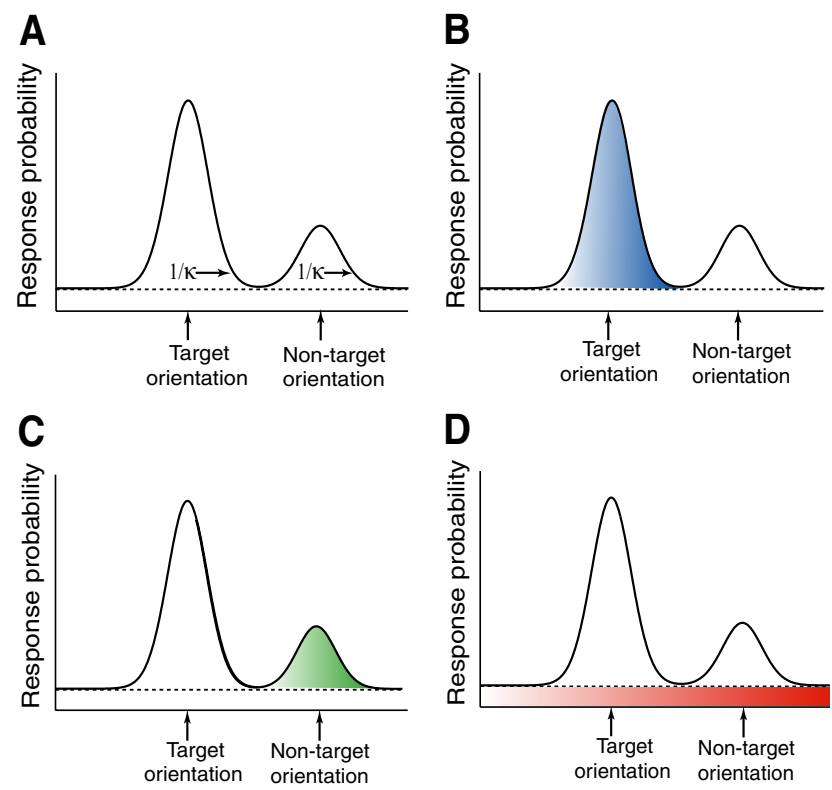

Figure 5. A probabilistic model of three sources of error in subjects' responses. Subject responses on the memory task were decomposed into three separate components, illustrated by the shaded regions in $\boldsymbol{B}-\boldsymbol{D}$ : a circular Gaussian distribution of responses centered on the orientation value of the target $(\boldsymbol{B})$; circular Gaussian distributions with the same width centered on each nontarget orientation value, corresponding to misbinding errors $(\boldsymbol{C})$; and a uniform distribution, capturing random responses unrelated to any of the sample orientations $(\boldsymbol{D})$. $\boldsymbol{A}$, The variability in recall of each item's orientation was governed by $\kappa$, the concentration parameter of the circular Gaussian (von Mises) distributions.

feature, independent of any particular model of the underlying response distribution. To investigate further possible mechanisms producing the loss of memory precision for earlier items in sequences, we applied to the data a probabilistic model that assumes three potential sources of error, as follows: (1) Gaussian variability in recall of the target orientation, (2) a certain probability of responding with the remembered orientation of a nontarget due to associating incorrectly, or misbinding, a target's color with the orientation of a nontarget, and (3) a certain probability of producing a random response not related to any of the orientations presented (for further details, see Materials and Methods, above) (Fig. 5). This analysis method has been used in previous studies, but only for simultaneously presented items (Bays et al., 2009, 2011; Fougnie et al., 2010).

First, we fit the model separately for each number of items, presented sequentially or simultaneously. As shown in Figure 6, for both sequential (black) and simultaneous presentation (red), as the number of items increased, responses centered on the target became increasingly variable, as indicated by a significant decrease in the concentration parameter $(\kappa)$ of their distribution (two-way ANOVA, set size $\times$ presentation mode, main effect of set size: $F_{(4,75)}=15.9, p<0.001$; interaction with mode of presentation: $F_{(4,75)}=0.33, p=0.86$ ) (Fig. $6 A$ ). Importantly, the variability of subjects' responses for the same number of items was indistinguishable whether presented sequentially or simultaneously (main effect of presentation mode: $\left.F_{(1,75)}=0.47, p=0.50\right)$.

In addition to the increase in variability, the proportion of responses attributed to report of the correct target item $(\alpha)$ declined significantly as set size increased in both sequences and simultaneous arrays (main effect of set size: $F_{(3,60)}=13.7, p<$ 0.001; interaction: $F_{(3,60)}=0.33, p=0.05$ ) (Fig. $6 B$ ). Here, however, a significant difference was observed between sequential
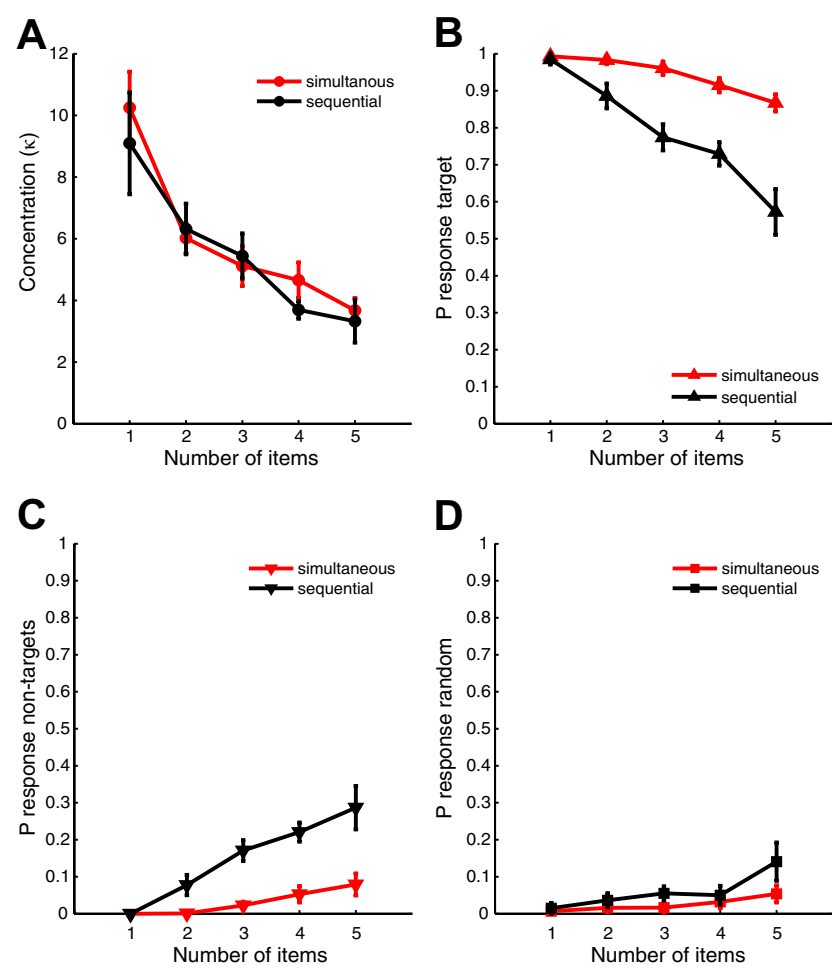

Figure 6. Model components for each set size in sequential and simultaneous presentation. $\boldsymbol{A}-\boldsymbol{D}$, Model parameters are shown for sequences (black) and simultaneous presentation (red). $A$, Variability of responses, as expressed by the concentration parameter of their distribution, increased as more items were stored in memory; this effect was not different between sequential and simultaneous presentation. $\boldsymbol{B}$, Probability of responding according to the target's orientation decreased with increasing set size and was lower in sequences. $\boldsymbol{C}$, There was a corresponding significant increase in the probability of responding according to a nontarget orientation; this component was greater in sequential than in simultaneous presentation. $\boldsymbol{D}$ Random responses (guessing) also increased with increasing number of items and were more likely in sequences. Note that, in sequences, the nontarget component $(\boldsymbol{C})$ was significantly higher than the random one $(\boldsymbol{D})$; this was not the case in simultaneous presentation, where there was no significant difference between these two components.

and simultaneous presentation (main effect of presentation mode: $\left.F_{(1,60)}=61.6, p<0.001\right)$, with a substantially smaller probability of responses centered on the target orientation under sequential presentation (mean $\alpha=74 \%$ for sequential vs mean $\alpha=93 \%$ for simultaneous presentation, for set size $\geq 2$ ).

The decline in the probability of responding with the target orientation with increasing set size coincided with a corresponding increase in both misreporting a nontarget as a target $(\beta$, main effect: $F_{(3,60)}=7.7, p<0.001$; interaction: $\left.F_{(3,60)}=1.6, p=0.21\right)$ (Fig. $6 C)$ and of responding with a random orientation $(\gamma$, main effect: $F_{(3,75)}=2.9, p=0.009$; interaction: $F_{(3,75)}=0.84, p=$ 0.50 ) (Fig. 6D). However, the difference between sequential and simultaneous presentation was primarily accounted for by changes in the rate of misreporting (mean $\beta=19 \%$ for sequential vs mean $\beta=4 \%$ for simultaneous presentation, for set size $\geq 2$; $\left.F_{(1,60)}=46.5, p<0.001\right)$.

Random responses were also significantly more probable in sequences $\left(F_{(1,75)}=5.0, p=0.028\right)$ but to a much smaller extent than nontarget responses (mean $\gamma=7 \%$ for sequential vs mean $\gamma=3 \%$ for simultaneous presentation, for set size $\geq 2$ ). It is important to note that in sequences of two or more items, misreporting accounts for a significantly greater proportion of the overall loss of precision when compared with random responses $\left(F_{(1,64)}=23.0, p<0.001\right)$. 
A
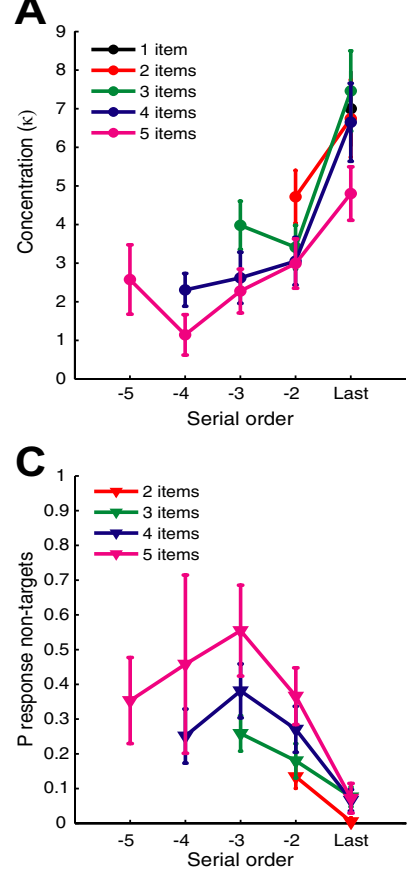

B

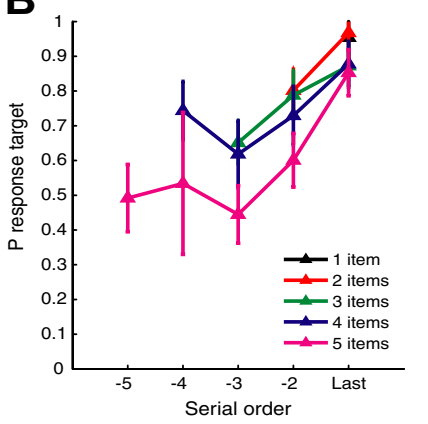

D

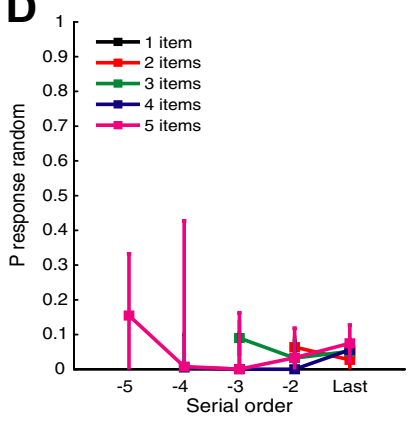

Figure 7. Model components for each serial order. $\boldsymbol{A}, \boldsymbol{B}$, Variability $(\boldsymbol{A})$ and probability $(\boldsymbol{B})$ of responding according to the target's orientation depend on serial order, with responses on target being more probable when the last item was probed. $\boldsymbol{C}$, There was a corresponding increase in the probability of responding according to a nontarget for items earlier than the last. $D$, Conversely, the probability of responding randomly was not significantly different for items with different order in the sequence. Error bars represent SEM.

To investigate whether the contribution of the different sources of error to the overall loss of precision depends on the item's order in the sequence, we applied the model separately at each serial position and each sequence length. As shown in Figure 7, $A$ and $B$, both the probability of reporting the target item's orientation and the variability of those responses vary according to serial order of an item in the sequence $\left(\alpha: \chi^{2}>13.5, p<0.003\right.$ for all sequence lengths; $\kappa: \chi^{2}>13.9, p<0.001$ for all sequence lengths except in two items, where $\left.\chi^{2}=2.8, p=0.096\right)$. Responses based on the correct target orientation were significantly more likely and less variable when the last item was probed $\left(\alpha: \chi^{2}\right.$ $>13.5, p<0.003$ for all sequence lengths; $\kappa: \chi^{2}>12.9, p<0.001$ for all sequence lengths except in two items, as above).

Correspondingly, the probability of responding according to the orientation of a nontarget depended significantly on the serial order of the tested item $\left(\chi^{2}>11.4, p<0.004\right.$ for all sequence lengths) and was less likely for the last item than for any of the previous ones ( $\chi^{2}>10.2, p<0.002$ for all sequence lengths) (Fig. $7 C$ ). Within the longest sequences (four or five items), from earlier to later items, the probability of misbinding increases and subsequently decreases again for more recent items, producing an inverted U-shaped curve. This suggests both a recency and a primacy effect in the probability of responding with the orientation of a nontarget (Fig. 7C).

The probability of responding randomly did not differ significantly with serial order. In sequences of any length, random responses were relatively rare (5.9\% on average) and unaffected by serial order $\left(\chi^{2}<1.3, p>0.37\right)$ (Fig. $\left.7 D\right)$. Overall, these results suggest that an increased probability of erroneously associating the color of targets earlier in the sequence with the orientation of nontargets could be an important mechanism giving rise

to the overall precision cost of sequential presentation, particularly for items in the middle of a sequence.

Our findings also demonstrate that information about different objects held in WM are not independent once stored, but can interact-hence misbinding of object features across visual items. In addition, the comparison between sequential and simultaneous displays reveals an important factor about how WM resources are dynamically reallocated. Recall that, for sequences, subjects did not know how many items they would have to remember before a trial commenced, so they could not preallocate resources. Instead, each new object to be remembered (the current last item) was allocated its fair share of resources (as if it had appeared in a simultaneous array of the same total number of items). However, the resolution in memory for previous items became inferior. This reallocation of resources from earlier items was associated specifically with increased misbinding of features belonging to different objects. These findings place very tight constraints on neural models of WM.

Note that, as the variability of responses and the number of nontargets increase, reliability of the fitted parameter estimates decreases, as indicated by the larger SE (e.g., set size four and five) (Fig. 7). In the case of two items, for example, a distribution of responses tightly centered around the only nontarget orientation is easily distinguishable from a set of random responses. Conversely, in the case of five items, where each misbinding response can correspond to any of four different nontarget orientations, distinguishing this from a uniform (random) distribution becomes increasingly difficult. However, while this unavoidable limitation reduces power, we were nonetheless able to identify statistically significant effects (e.g., of serial order) even in the longest sequences.

\section{Simultaneous versus sequential presentation at different spatial locations}

The comparison between simultaneous and sequential presentation in the previous experiments may have been confounded by the fact that sequentially presented items were shown at the same spatial location, which was not the case for simultaneous presentation. In experiment 3, we presented every object at a different location, in either simultaneous or sequential display.

As shown in Figure 8, average memory precision for items presented sequentially at different locations (black line) was significantly lower than precision for the same number of items presented simultaneously (repeated-measures ANOVA: $F_{(1,7)}=$ 29.94, $p=0.001$ ) (red line), in keeping with our results from experiments 1 and 2. Also consistent with our previous results, precision for the most recent object in a sequence of items shown at different locations (Fig. 8, blue dotted line) was no different from the average precision when the same number of objects were presented simultaneously (repeated-measures ANOVA: $\left.F_{(1,7)}=0.33, p=0.58\right)$. Therefore, the finding that memory precision is lower for sequential compared with simultaneous presentation cannot be simply attributed to spatial overwriting of earlier items, as precision is lower than simultaneous presentation also when each item in the sequence is presented at a different location.

Next, to quantify the parameters explaining the loss of memory resolution when objects are presented sequentially at different locations, we applied our probabilistic model analysis to the data from experiment 3. Consistently with our previous results, while the variability of responses was similar to simultaneous presentation $\left(\kappa: F_{(1,7)}=0.62, p=0.457\right)$ (Fig. 9A), responses to the target orientation were significantly less likely in sequential 


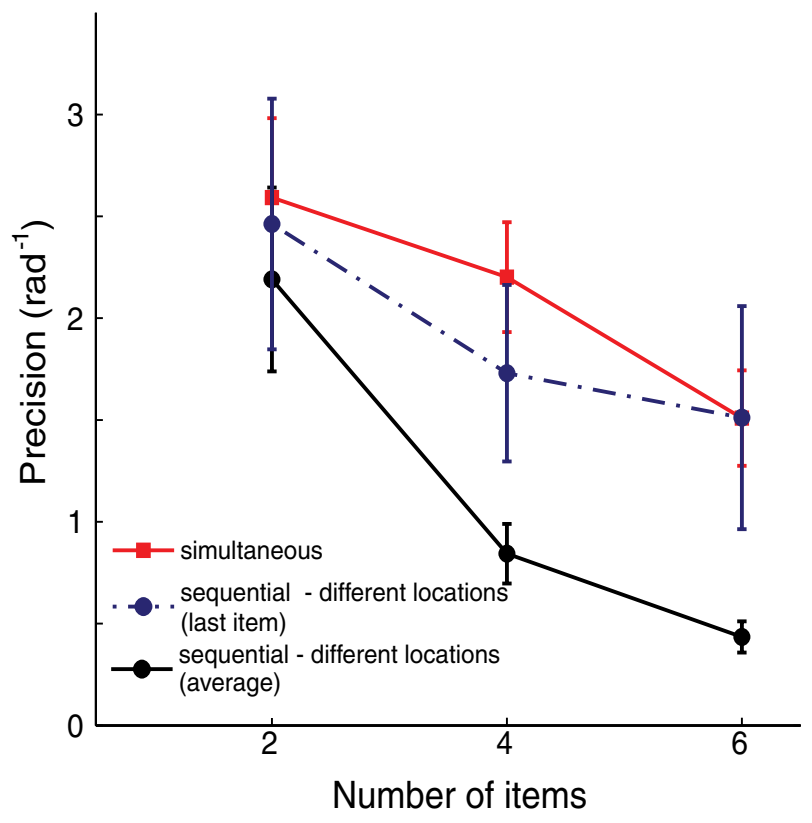

Figure 8. Comparing simultaneous and sequential presentation when all items are displayed at different locations. When each object in a sequence was presented at a different spatial location, memory across all items in the sequence (black line) was, on average, less precise than when the same number of objects was presented simultaneously (red line). Precision for the last item in a sequence (blue dashed line) is no different than for an item in an array of the same set size. Note that, for both simultaneous and sequential presentation, precision values are similar to those in Figure 3. Error bars are SEM.
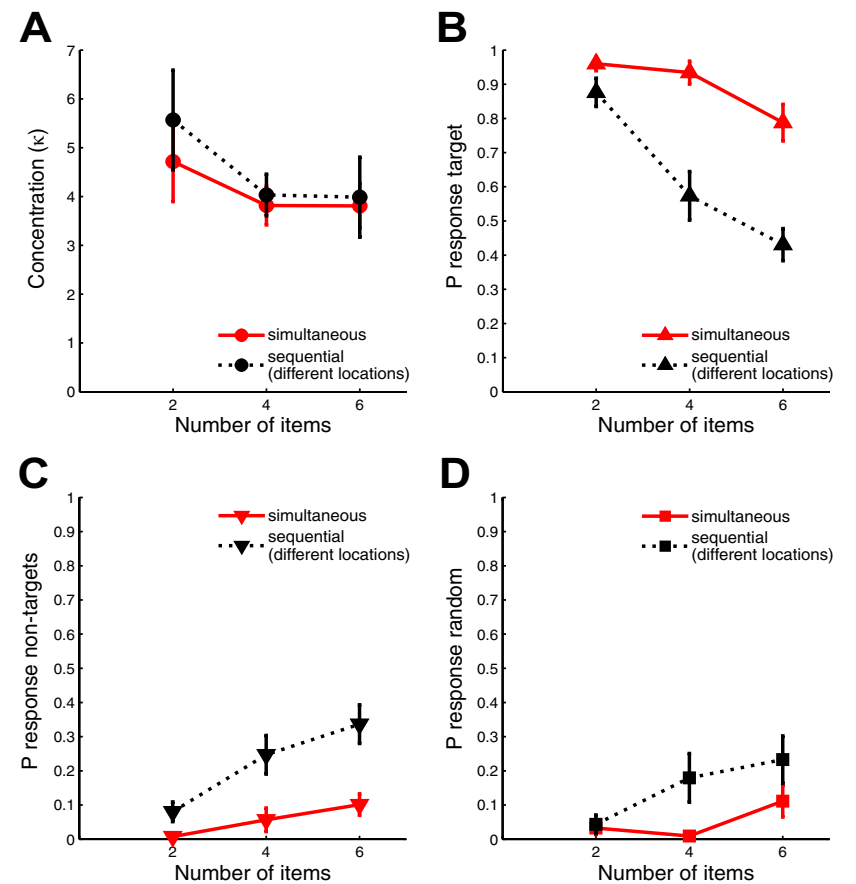

Figure 9. Model components for simultaneous and sequential presentation, when all items are displayed at different locations. Model parameters are shown for items displayed sequentially at different locations (dotted black line) and for simultaneous presentation (red line). $\boldsymbol{A}$, The variability of responses was not different between the presentation modes. $\boldsymbol{B}$, The probability of responding to the target orientation was significantly lower for sequential presentation. This can be explained by an increase in nontarget responses (due to misbinding of the target color and a nontarget orientation) in the case of sequential presentation $(\boldsymbol{C})$ and, to a lesser extent, by an increase in random responses (D).
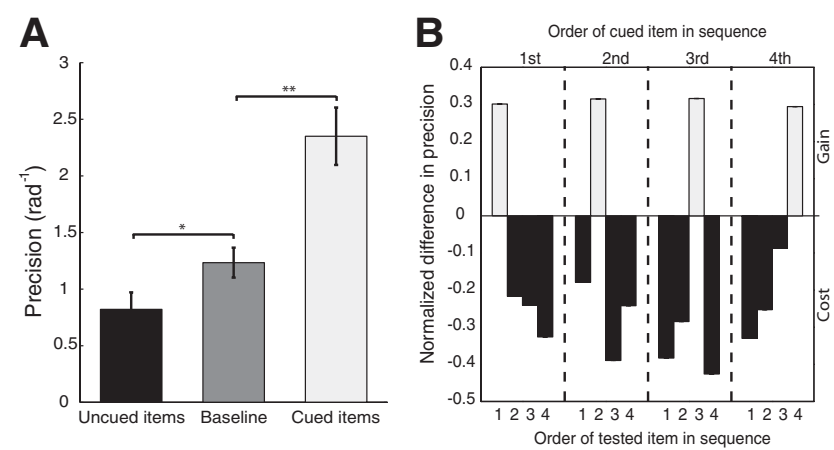

Figure 10. Memory precision depends on task relevance. $\boldsymbol{A}$, In the trials where a cue was present, the cued item was remembered significantly better than items in a baseline condition where no cue was present, with a corresponding cost in memory precision for the less taskrelevant items. Error bars are SEM. ${ }^{* *} p<0.001,{ }^{*} p<0.01$. B, Fractional difference in precision between trials where a cue was present and the baseline condition. Gain in memory precision for cued items (light gray) and a cost for the uncued ones (dark gray) was observed at all possible serial positions of the cued and the tested item.

presentation $\left(\alpha: F_{(1,7)}=47.4, p<0.001\right)$ (Fig. $\left.9 B\right)$. Importantly, a significant proportion of the loss in memory resolution for sequentially presented items can be attributed to nontarget responses (due to misbinding between visual features of different objects in the sequence) also when each object is projected at a different spatial location $\left(\beta: F_{(1,7)}=13.42, p=0.008\right)$ (Fig. 9C). Therefore, increased misbinding in sequences does not occur only when the spatial locations of the misbound objects overlap. As previously stated, we also note a significant increase in random responses in sequences when compared with simultaneous presentation $\left(\gamma: F_{(1,7)}=6.48, p=0.038\right)($ Fig. 9D).

\section{Memory precision in sequences depends on task relevance}

In everyday experience of the visual world, objects perceived across a period of time are rarely equally important or equally relevant to the task at hand. Therefore, it is crucial to examine how memory precision is affected by the relative behavioral relevance or priority of each of the objects in a sequence.

In experiment 4 , we displayed four items sequentially and made one of them more task-relevant by increasing its relative probability to be tested. This prioritized, or cued, item was always of the same color and subjects were informed that one color was more likely to be tested than the others. We investigated memory precision for this cued item and also for the remaining, uncued items in the sequence. Performance in this task was compared with a neutral baseline condition, where all four items were equally probable to be tested. As shown in Figure $10 \mathrm{~A}$, memory for the cued item was significantly more precise than baseline $\left(t_{(8)}=5.8\right.$, $p<0.001$ ), with a significant corresponding cost for the uncued objects $\left(t_{(8)}=3.5, p=0.008\right)$.

We also investigated whether the benefit of cueing on memory precision - and the cost to the uncued items - are evenly distributed across the sequence, with respect to the serial order of the prioritized item. More specifically, we asked whether the relative benefit of cueing depends on serial order and whether the cost is distributed to all of the uncued items or, alternatively, whether it is limited to those preceding or those following the cued item. Therefore, for every item in the sequence, we calculated the fractional difference in precision between the baseline condition and the cue present condition for each possible serial order of the cued item. Positive values of this measure signify a gain and negative values a cost in memory precision when compared with the baseline condition. As shown in Figure $10 B$, the relative gain in 
A

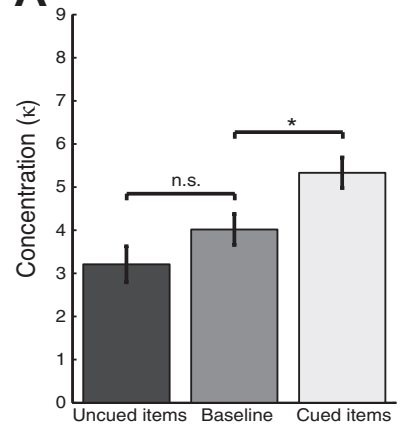

C

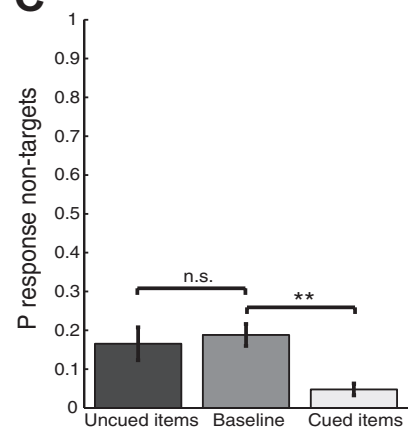

B

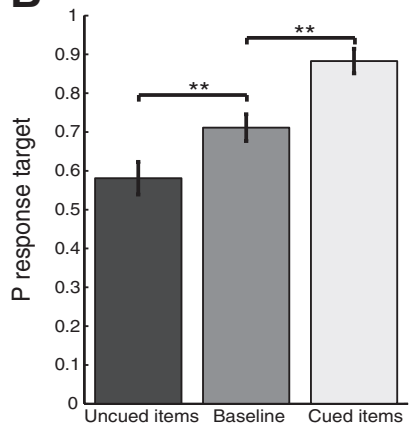

D

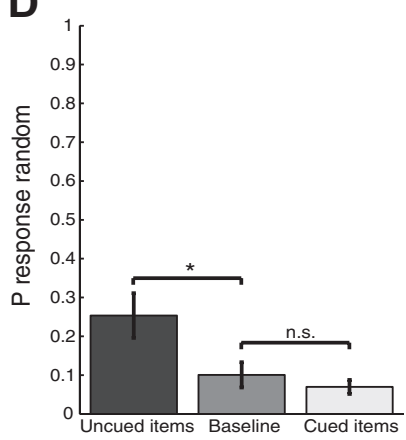

Figure 11. Model components in relation to task relevance. $\boldsymbol{A}$, Responses centered at the target orientation were less variable for cued item when compared with baseline. There was a corresponding nonsignificant increase in variability for the remaining, less task-relevant items. $\boldsymbol{B}$, The probability of responding according to the target's orientation was enhanced for cued items, with a corresponding cost for uncued ones. C, Probability of responding according to a nontarget orientation was lower for the more task-relevant item, while this parameter was no different from baseline for the uncued items. $\boldsymbol{D}$, Conversely, random responses were increased for uncued items and were similar to baseline for the cued item. Error bars represent SEM. ${ }^{* *} p<0.001,{ }^{*} p<0.05 ;$ n.s., nonsignificant.

precision was similar for the cued items, regardless of their order in the sequence, and the relative cost was distributed between all of the uncued items, both those preceded by the cued item and those followed by it.

Next, we sought to identify the sources of error resulting in decreases in memory precision for the less task-relevant items and the corresponding benefit for the cued ones. To this end, using the probabilistic model described above, we estimated the Gaussian variability of the responses to the target, the probability of responding according to a nontarget, and the probability of responding with a random orientation separately for the cued and noncued items (in the condition where a cue was present) and for the baseline condition where all items were equally task relevant.

The results are presented in Figure 11. Gaussian variability for the cued item is significantly less than for an uncued item in the same sequence $\left(\chi^{2}=13.5, p=0.001\right)$ or an item in the condition where cueing is not present $\left(\chi^{2}=5.7, p=0.017\right)($ Fig. 11A). The probability of responding with the target orientation is significantly higher for the cued items when compared with baseline $\left(\chi^{2}=33.1, p<0.001\right)$ (Fig. $\left.11 B\right)$, with a corresponding decrease in target responses for the uncued items $\left(\chi^{2}=8.1, p=0.004\right)$ (Fig. $9 B$ ). As shown in Figure $11 C$, a significant part of the gain in overall precision for the cued items can be attributed to a reduction in the probability of misreporting another item's orientation compared with uncued items $\left(\chi^{2}=23.8, p=0.017\right)$ or baseline $\left(\chi^{2}=20, p=0.018\right)$. In other words, increasing the task relevance of an item seems to facilitate successful binding between its

visual features. The corresponding decrease in memory resolution for the less task-relevant items is, to a significant extent, due to an increase in random responses $\left(\chi^{2}=5.3, p=0.022\right)$ (Fig. $11 D)$ rather than an increase in misbinding, the probability of which is similar to the baseline condition $\left(\chi^{2}=0.1, p=0.66\right)$ (Fig. 11D).

\section{Discussion}

We examined the fidelity of visual WM for orientation of objects presented in sequence by analyzing precision of observers' reports, rather than asking them about the presence or absence of a change (Luck and Vogel, 1997; Vogel et al., 2001). There was a marked decrease in precision as the number of items to be remembered increased (Figs. 2, 3). Thus, memory capacity is indeed highly limited. Importantly, however, WM resolution decreased smoothly as total number of items increased (Fig. 3), and this loss of fidelity affected every item in the sequence (Fig. 2). Even adding a single item to a previous object held in memory was sufficient to produce a significant drop in mean precision of report (Fig. 3).

These data cannot be adequately explained by a simple slot model in which WM is limited to three or four items because this would predict optimal performance until the object capacity limit is reached, and a sharp drop in precision when that limit is exceeded (Pashler, 1988; Luck and Vogel, 1997; Cowan, 2001). Instead, our results are compatible with the concept of a limited memory resource, a proportion of which is allocated to each item as the total number of items increases. Such a proposal also provides a parsimonious account of WM limits in the case of simultaneously presented objects (Bays and Husain, 2008; Bays et al., 2009).

Measuring WM precision revealed in addition that, although there is a recency effect for all sequence lengths, the fidelity of memory for the last item was strongly modulated by the number of items that preceded it (Fig. 2). Precision for the last item was worse in longer sequences, a finding that has implications for neural models of serial WM. Previous studies using similar display intervals also reported recency effects (Phillips and Christie, 1977; Broadbent and Broadbent, 1981; Wright et al., 1985; Neath, 1993; Hay et al., 2007; Blalock and Clegg, 2010), but none showed that last-item recency is affected by sequence length in this way. Again, those studies used binary measures of report (correct or incorrect); precision, we argue, provides a more sensitive index that better constrains models of WM and their neurophysiological substrates.

Comparison of sequential versus simultaneous arrays (Fig. 3) demonstrated that, while precision in both cases fell smoothly with increasing number of objects, mean precision across all items was significantly worse for sequences than for simultaneous arrays (Lecerf and De Ribaupierre, 2005; Allen et al., 2006; Blalock and Clegg, 2010). Crucially, however, this cost of sequential presentation was restricted to items preceding the last object. Thus, for example, the last item in a sequence of four objects was recalled with the same precision as if it had been tested in an array of four items presented simultaneously, while all other items in the sequence were recalled less precisely. This cannot be accounted for by temporal decay (Fig. 4), consistent with studies of serial verbal WM (Lewandowsky et al., 2009). Furthermore, we show that lower precision in sequential versus simultaneous presentation is not due to spatial overwriting, as it is replicated when all items are presented sequentially at different locations (Fig. 8). So how can we explain this effect? 
To address this question, we fit a probabilistic model (Fig. 5) to the distribution of recall errors that was previously developed for simultaneous presentation (Bays et al., 2009, 2011). Here, the analysis revealed that the Gaussian variability in recalling an item's orientation was, on average, equivalent whether the set of items was presented sequentially or simultaneously (Fig. 6A). Hence, the loss of overall fidelity observed with sequential presentation is not due to increased variability in storing each item's orientation.

However, in addition to accurate recall of orientation, successful performance also required accurate binding of orientation information with color. A seminal study suggested that different visual features are stored independently but it is their integration that is vulnerable to interference (Wheeler and Treisman, 2002). Memory for bound objects in a sequence is also more susceptible to interference from subsequent items than WM for individual features (Allen et al., 2006).

Our findings show that the probability of errors due to misbinding target color with the orientation of a nontarget was significantly higher for sequences than for simultaneous presentation (Fig. 6C). Furthermore, these errors were more common for objects earlier in the sequence than for the last item (Fig. 7C). It has been shown previously that spatial location has a central role in feature binding (Treisman and Zhang, 2006). Our results show that increased misbinding in sequential presentation also occurs when the locations of misbound objects do not overlap (Fig. 9C).

The performance cost observed when comparing sequential to simultaneous presentation is primarily due to an increased probability of misbinding when items are presented in a sequence. Thus, there is interference across stored representations, a phenomenon that is also not predicted by independent object slots. A smaller but significant proportion of responses were attributed to a random component, which could correspond to simple guessing. However, this did not change significantly between the last and preceding items (Fig. 7D), and so cannot account for the performance cost specific to earlier items.

While variability in the Gaussian component of error for items presented sequentially was, on average, equivalent to that observed in simultaneous presentation, when comparing this parameter between items in different serial orders, there was less variability in recalling orientation of the last item than previous ones (Fig. 7A). Thus, whereas each object in a simultaneously presented array theoretically is allocated the same amount of WM resource, this is clearly not the case in sequences where each new object to be remembered (the current last item) was allocated its fair share of resources (as if it had appeared in a simultaneous array of the same total number of items). However, the resolution in memory for previous items became inferior.

Thus, there is a dynamic redistribution of memory resource in sequences; this reallocation from earlier items was associated specifically with increased misbinding of features belonging to different objects. While we conceptualize this process to be the shift of internal resources (Chun et al., 2011) from items already held in memory to a newly added item, there are alternative possibilities.

First, it might be argued that it is possible for a memory slot to abandon the object it currently holds and switch to maintaining a new object. Critically, the revised slot model (Zhang and Luck, 2008) predicts fixed-resolution memory representations that would be difficult to reconcile with some of the findings of the current study. For example, if the most recent item displaces a previous item from a fixed-resolution slot, responses for one or more items in the previous serial positions should be either equally accurate to those for the last item (when a previous item keeps its slot) or consistently below chance (when a previous item is left without a slot). But our findings suggest neither is the case, even if we assume that two or more slots double-up (Zhang and Luck, 2008) to accommodate the last item with higher precision than the previous objects.

Second, it might be argued that allocation of greater attention (i.e., external resources) to the most recent item, plus increasing passive interference between items held in memory as new items are added, might also explain our findings. However, such a model would need to posit some separation between (internal) resources for working memory and extra (external) resources available for attention, an area of research that remains to be resolved (for discussion, see Chun et al., 2011). Moreover, this still remains an updating of resources, as attention is redeployed to the new item. Our experiments with cuing an object demonstrated that greater attention to the target leads to improved precision of recall but with a concurrent cost to other items.

Interestingly, the increase in memory resolution with cuing was explained by a decrease both in the variability of storing its orientation and in the probability of misbinding its color with a nontarget's orientation (Fig. 9), suggesting that the bound representation of a prioritized object early in the sequence was more resistant to interference from subsequent, less task-relevant ones. The corresponding drop in precision for less task-relevant objects also extended to items at any order in the sequence, but could not be attributed to increased misbinding. Instead, there was a significant increase in the random error component for report of these items, suggesting they were not so accurately encoded. Recent studies demonstrated that, in simultaneous presentation, individual WM differences are determined by processes such as attentional selection, taking place during encoding rather than during maintenance in memory (Cusack et al., 2009; Linke et al., 2011). Future studies should investigate the ability to prioritize task-relevant items in sequences in the context of individual differences.

Finally, although in the current study we investigated WM for one visual dimension (orientation), a recent study of sequential WM for visual motion showed similar results (Zokaei et al., 2011). Thus, the principles discussed here are not confined to only one feature.

In summary, using precision as an index of WM provides important new insights into the nature of memory representations of objects viewed at different times, revealing how resources can be dynamically and flexibly reallocated and providing a credible framework for interpreting neural data.

\section{References}

Allen RJ, Baddeley AD, Hitch GJ (2006) Is the binding of visual features in working memory resource-demanding? J Exp Psychol Gen 135:298-313.

Alvarez GA, Cavanagh P (2004) The capacity of visual short-term memory is set both by visual information load and by number of objects. Psychol Sci 15:106-111

Baddeley A (2003) Working memory: looking back and looking forward. Nat Rev Neurosci 4:829-839.

Bays PM, Husain M (2008) Dynamic shifts of limited working memory resources in human vision. Science 321:851-854.

Bays PM, Catalao R, Husain M (2009) The precision of visual working memory is set by allocation of a shared resource. J Vis 9:7.1-7.11

Bays PM, Wu EY, Husain M (2011) Storage and binding of object features in visual working memory. Neuropsychologia 49:1622-1631.

Berman MG, Jonides J, Lewis RL (2009) In search of decay in verbal shortterm memory. J Exp Psychol Learn Mem Cogn 35:317-333.

Blalock LD, Clegg BA (2010) Encoding and representation of simultaneous 
and sequential arrays in visuospatial working memory. Q J Exp Psychol 63:856-862.

Broadbent DE, Broadbent MH (1981) Recency effects in visual memory. Q J Exp Psychol 33:1-15.

Burgess N, Hitch GJ (1999) Memory for serial order: a network model of the phonological loop and its timing. Psychol Rev 106:551-581.

Chun MM, Golomb JD, Turk-Browne NB (2011) A taxonomy of external and internal attention. Annu Rev Psychol 62:73-101.

Cowan N (2001) The magical number 4 in short-term memory: a reconsideration of mental storage capacity. Behav Brain Sci 24:87-114.

Cowan N (2005) Working memory capacity. New York: Psychology.

Cusack R, Lehmann M, Veldsman M, Mitchell DJ (2009) Encoding strategy and not visual working memory capacity correlates with intelligence. Psychon Bull Rev 16:641-647.

D'Esposito M, Postle BR, Jonides J, Smith EE (1999) The neural substrate and temporal dynamics of interference effects in working memory as revealed by event-related functional MRI. Proc Natl Acad Sci U S A 96:7514-7519.

Fisher NI (1993) Statistical analysis of circular data. Cambridge: Cambridge UP.

Fougnie D, Asplund CL, Marois R (2010) What are the units of storage in visual working memory? J Vis 10:27.

Hay DC, Smyth MM, Hitch GJ, Horton NJ (2007) Serial position effects in short-term visual memory: a SIMPLE explanation? Mem Cognit 35:176-190.

Hole GJ (1996) Decay and interference effects in visuospatial short-term memory. Perception 25:53-64.

Jenkins LJ, Ranganath C (2010) Prefrontal and medial temporal lobe activity at encoding predicts temporal context memory. J Neurosci 30:15558-15565.

Johnson AJ, Miles C (2009) Serial position effects in 2-alternative forced choice recognition: functional equivalence across visual and auditory modalities. Memory 17:84-91.

Lecerf T, De Ribaupierre A (2005) Recognition in a visuospatial memory task: the effect of presentation. Eur J Cogn Psychol 17:47-75.

Lewandowsky S, Oberauer K, Brown GD (2009) No temporal decay in verbal short-term memory. Trends Cogn Sci 13:120-126.

Linke AC, Vicente-Grabovetsky A, Mitchell DJ, Cusack R (2011) Encoding strategy accounts for individual differences in change detection measures of VSTM. Neuropsychologia 49:1476-1786.

Logie RH, Della Sala S, Wynn V, Baddeley AD (2000) Visual similarity effects in immediate verbal serial recall. Q J Exp Psychol A 53:626-646.

Luck SJ, Vogel EK (1997) The capacity of visual working memory for features and conjunctions. Nature 390:279-281.

Marshuetz C, Smith EE, Jonides J, DeGutis J, Chenevert TL (2000) Order information in working memory: fMRI evidence for parietal and prefrontal mechanisms. J Cogn Neurosci 12:130-144.
Myung IJ (2003) Tutorial on maximum likelihood estimation. J Math Psychol 47:90-100.

Neath I (1993) Distinctiveness and serial position effects in recognition. Mem Cognit 21:689-698.

Palmer J (1990) Attentional limits on the perception and memory of visual information. J Exp Psychol Hum Percept Perform 16:332-350.

Pashler H (1988) Familiarity and visual change detection. Percept Psychophys 44:369-378.

Phillips WA (1974) On the distinction between sensory storage and shortterm visual memory. Percept Psychophys 16:283-290.

Phillips WA, Christie DF (1977) Components of visual memory. Q J Exp Psychol 29:117-133.

Siegel M, Warden MR, Miller EK (2009) Phase-dependent neuronal coding of objects in short-term memory. Proc Natl Acad Sci USA 106: 21341-21346.

Smyth MM, Hay DC, Hitch GJ, Horton NJ (2005) Serial position memory in the visual-spatial domain: reconstructing sequences of unfamiliar faces. Q J Exp Psychol A 58:909-930.

Takahama S, Miyauchi S, Saiki J (2010) Neural basis for dynamic updating of object representation in visual working memory. Neuroimage 49:3394-3403.

Todd JJ, Marois R (2004) Capacity limit of visual short-term memory in human posterior parietal cortex. Nature 428:751-754.

Treisman A, Zhang W (2006) Location and binding in visual working memory. Mem Cognit 34:1704-1719.

Vogel EK, Woodman GF, Luck SJ (2001) Storage of features, conjunctions and objects in visual working memory. J Exp Psychol Hum Percept Perform 27:92-114.

Warden MR, Miller EK (2010) Task-dependent changes in short-term memory in the prefrontal cortex. J Neurosci 30:15801-15810.

Wheeler ME, Treisman AM (2002) Binding in short-term visual memory. J Exp Psychol 131:48-64.

Wilken P, Ma WJ (2004) A detection theory account of change detection. J Vis 4:1120-1135.

Wright AA, Santiago HC, Sands SF, Kendrick DF, Cook RG (1985) Memory processing of serial lists by pigeons, monkeys, and people. Science 229:287-289.

Xu Y, Chun MM (2006) Dissociable neural mechanisms supporting visual short-term memory for objects. Nature 440:91-95.

Zhang W, Luck SJ (2008) Discrete fixed-resolution representations in visual working memory. Nature 453:233-235.

Zhang W, Luck SJ (2009) Sudden death and gradual decay in visual working memory. Psychol Sci 20:423-428.

Zokaei N, Gorgoraptis N, Bahrami B, Bays PM, Husain M (2011) Visual working memory for motion sequences. Paper presented at Vision Sciences Society 11th Annual Meeting, Naples, Florida, May. 